\title{
Prevalência de tentativas de suicídio entre adolescentes e jovens*
}

\author{
Erasmo de Carvalho Oliveira ${ }^{1}$ \\ (D) https://orcid.org/0000-0003-0579-1297 \\ Tatiane Scolastrici Meucci ${ }^{1}$ \\ (D) https://orcid.org/0000-0001-9228-5872 \\ Lisabelle Mariano Rossato² \\ (D) https://orcid.org/0000-0003-3375-8101 \\ Ana Márcia Chiaradia Mendes-Castillo ${ }^{3}$ \\ (D) https://orcid.org/0000-0002-0367-1606 \\ Lucía Silva ${ }^{4}$ \\ (DD https://orcid.org/0000-0002-6353-7580
}

\footnotetext{
* Este artigo refere-se à chamada temática "Violência autoprovocada: autolesão não suicida e comportamento suicida".

1 Universidade Nove de Julho, Faculdade Marechal Rondon, São Manuel, SP, Brasil.

2 Universidade de São Paulo, Escola de Enfermagem, São Paulo, SP, Brasil.

${ }^{3}$ Universidade Estadual de Campinas, Faculdade de Enfermagem, Campinas, SP, Brasil.

${ }^{4}$ Universidade Federal de São Paulo, Escola Paulista de Enfermagem, São Paulo, SP, Brasil.
}

Objetivo: caracterizar as tentativas de suicídio entre adolescentes e jovens em um município brasileiro. Método: trata-se de estudo quantitativo, retrospectivo, descritivo exploratório, realizado no Núcleo de Apoio à Saúde da Família. Foram coletadas informações de 55 fichas de notificação compulsória de pessoas entre 12 e 25 anos de idade, notificadas como tentativa de suicídio pelo Núcleo de Vigilância Epidemiológica, no período estudado. A organização e a análise dos dados foram realizadas por meio de estatística descritiva. Resultados: evidenciou-se uma amostra composta principalmente por pessoas de 22 a 25 anos de idade, mulheres, da raça branca, estudantes, solteiras, residentes em zona urbana, com tentativa de suicídio no próprio domicílio, no período noturno, por envenenamento. Conclusão: este perfil assemelha-se ao perfil das tentativas entre adultos, apontado na literatura, sinalizando a importância de os profissionais programarem estratégias de prevenção inclusive entre crianças e adolescentes, a fim de se evitar, futuramente, não somente as tentativas, mas também o suicídio entre essa população.

Descritores: Tentativa de Suicídio; Suicídio; Envenenamento; Morte; Violência; Adolescente.

\section{Como citar este artigo}

Oliveira EC, Meucci TS, Rossato LM, Mendes-Castillo AMC, Silva L. Prevalence of suicide attempts among adolescents and young people. SMAD, Rev Eletrônica Saúde Mental Álcool Drog. 2020;16(4):85-91. doi: https://dx.doi.org/10.11606/issn.1806-6976.smad.2020.168441 


\section{Prevalence of suicide attempts among adolescents and young people}

Objective: to characterize the suicide attempts among adolescents and young people in a Brazilian city. Method: this is a quantitative, retrospective, exploratory, and descriptive study, carried out at the Family Health Support Center. Data were collected from 55 files of compulsory notification of individuals between 12 and 25 years old, notified as suicide attempt by the Center for Epidemiological Surveillance, during the study period. The organization and analysis of the data were performed by means of descriptive statistics. Results: the sample was mainly composed of individuals aged 22 to 25 years old, white-skinned women, students, single women, living in urban areas, with suicide attempted at home, at night, by poisoning. Conclusion: this profile is similar to the profile of attempts among adults highlighted in the literature, indicating the importance for the professionals to implement prevention strategies also among children and adolescents, in order to avoid not only attempts but also suicide among this population.

Descriptors: Suicide Attempted; Suicide; Poisoning; Death; Violence; Adolescent.

\section{Prevalencia de intentos de suicidio entre adolescentes y jóvenes}

Objetivo: caracterizar los intentos de suicidio entre adolescentes y jóvenes en un municipio brasileño. Método: estudio exploratorio cuantitativo, retrospectivo, descriptivo, realizado en el Centro de Apoyo a la Salud de la Familia. Se recopiló información de 55 registros de notificación obligatoria de personas entre 12 y 25 años, notificadas como intento de suicidio por el Centro de Vigilancia Epidemiológica, durante el período estudiado. La organización y el análisis de los datos se realizaron con estadística descriptiva. Resultados: se compuso una muestra principalmente de personas de 22 a 25 años, mujeres, blancas, estudiantes, solteras, que viven en áreas urbanas, con intento de suicidio en el hogar, por la noche, por envenenamiento. Conclusion: este perfil es similar al perfil de los intentos entre adultos, señalado en la literatura, lo que indica la importancia de los profesionales para implementar estrategias de prevención incluso entre niños y adolescentes, para evitar, en el futuro, no solo intentos, sino también suicidios entre esta población.

Descriptores: Intento de Suicidio; Suicidio; Envenenamiento; Muerte; Violencia; Adolescente. 


\section{Introdução}

No Brasil, apesar de o Estatuto da Criança e do Adolescente definir como adolescentes os indivíduos entre 12 e 18 anos incompletos e, o Estatuto da Juventude considerar como jovens aqueles com idade entre 15 e 19 anos, as políticas públicas brasileiras mais recentes sinalizam os limites da faixa etária entre 10 e 24 anos para as ações prioritárias em saúde, mesmo nas recomendações específicas para adolescentes ${ }^{(1)}$.

O Ministério da Saúde, gestor federal do Sistema Único de Saúde (SUS) no Brasil, recebeu a determinação específica do Estatuto da Criança e do Adolescente (ECA) para promover o direito à vida e à saúde dessa população. No entanto, por vezes, o alcance da saúde integral desses indivíduos é ameaçado pela violência(1).

O suicídio é um tipo de violência que acomete todas as faixas etárias, incluindo adolescentes e jovens, sendo classificado como violência autoprovocada, intencionalmente, no sentido de o indivíduo terminar com sua própria vida, sendo considerado um problema de saúde pública, em todo o mundo. Já a tentativa de suicídio diferencia-se pelo desfecho, que não ocasiona a morte após a tentativa(2).

No Brasil, foram notificados 55.649 óbitos por suicídio entre 2011 e 2015, representando uma taxa de 5,5/100.000 habitantes. Já especificamente em relação às tentativas de suicídio, foco do presente estudo, foram registradas 48.204 ocorrências de 2011 a 2016, no Sistema de Informação de Agravos de Notificação (Sinan), sendo que $25,9 \%$ dos casos acometeram o sexo feminino e $19,6 \%$ acometeram o sexo masculino, entre pessoas de 10 a $19 \operatorname{anos}^{(3)}$.

No Brasil, observa-se aumento das taxas de suicídio entre adolescentes e jovens, representando a terceira maior causa de morte entre a população masculina na faixa etária de 15 a 29 anos e a oitava maior causa de mortalidade entre a população feminina que se encontra na mesma faixa etária(4). Além do Brasil, este aumento também vem sendo sinalizado em estudos realizados em diversos países, como China(5), África do Sul(6), França(7), Estados Unidos( ${ }^{(8)}$ e Peru ${ }^{(9)}$.

Este panorama mundial, que sinaliza o aumento das taxas de suicídio entre crianças e adolescentes, é muito preocupante, pois aponta uma direção contrária(10) ao cumprimento do acordo estabelecido pela Organização Mundial da Saúde, no sentido de reduzir as taxas de suicídio em até $10 \%$ até o ano de $2020^{(2)}$.

Outro ponto importante a ser considerado é o fato de que a maior parte dos adultos com transtornos mentais relatam seus primeiros sintomas de quadros depressivos ou ansiosos antes dos 24 anos de idade ${ }^{(11)}$.

Reconhecer que os mais jovens apresentam este tipo de sofrimento mental, assim como sofrimento que pode estar relacionado a fatores familiares, sociais, econômicos, culturais e individuais ${ }^{(10)}$, que os expõem a maior risco de comportamento suicida, é essencial para as políticas de prevenção. Um estudo que teve como objetivo conhecer os principais diagnósticos em um ambulatório geral que realiza consultas médicas e de enfermagem a adolescentes com problemas de saúde, entre 10 e 18 anos de idade, em Portugal, evidenciou que os transtornos mentais e de comportamento foram os mais prevalentes entre o grupo das meninas e entre o grupo dos adolescentes de 14 a 18 anos. As doenças mentais representaram o segundo grupo de diagnósticos mais prevalentes entre os adolescentes acompanhados por este ambulatório, sinalizando a importância de oferta de cuidado em saúde específico para esta população(12).

Fatores estressores como desentendimentos recentes com colegas, mau desempenho escolar, ruptura de relacionamentos afetivos ${ }^{(13)}$ e insatisfação relacionada ao excesso de peso e à magreza(14) estão associados com o risco elevado para o desenvolvimento de comportamento suicida entre adolescentes e jovens.

Outros estudos demonstram existir relação inversa entre o comportamento suicida dessa população e fatores de proteção familiar como apoio, coesão e adaptação na família diante de momentos de crise e adversidades. Nesse sentido, fatores de risco relacionados à estrutura e ao funcionamento familiar podem ser preditivos de tentativas e de suicídio nessa população(7,9,13).

Diante do conteúdo apresentado, destaca-se a importância do cuidado profissional aos transtornos mentais em adolescentes e jovens, diminuindo o risco de mortalidade e morbidade futura(11).

Nesse sentido, acredita-se que, conhecendo o perfil de adolescentes e jovens que realizam tentativas de suicídio, os profissionais da saúde podem reconhecer aqueles que se encontram sob maior risco, traçando estratégias de prevenção e intervenção mais contextualizadas, encaminhando esses jovens para cuidados especializados.

Portanto, o objetivo deste estudo foi caracterizar as tentativas de suicídio entre adolescentes e jovens em um município do interior paulista.

\section{Método}

Trata-se de estudo de natureza quantitativa, do tipo retrospectivo, descritivo exploratório, realizado no Núcleo de Apoio à Saúde da Família (NASF), estabelecido em uma unidade de saúde que abriga diversos serviços que oferecem apoio e qualificação para a rede de atenção primária à saúde da Secretaria Municipal de Saúde de Botucatu, município localizado na região centro oeste do Estado de São Paulo, Brasil.

Os dados foram coletados de abril de 2015 a fevereiro de 2016, por estudantes de graduação em enfermagem de uma universidade privada, localizada no 
município de São Manuel/SP, que dista cerca de $20 \mathrm{~km}$ de Botucatu, sob supervisão docente, mediante aprovação do Comitê de Ética em Pesquisa, via Plataforma Brasil (CAAE: 21828813.2.0000.5411).

Ressalta-se que os estudantes envolvidos com a coleta de dados deste estudo participavam de um projeto de extensão universitária na área de prevenção de suicídio. Conhecer o perfil das tentativas, apresentado neste artigo, bem como dos suicídios envolvendo adolescentes e jovens, foi uma das estratégias utilizadas a fim de aproximálos deste cenário, para sustentar o planejamento do processo de cuidar das famílias enlutadas(15), realizado posteriormente e também fomentar as discussões junto à equipe do NASF em relação a estratégias de prevenção de suicídio entre crianças e adolescentes, no âmbito da atenção primária à saúde.

Para tal, uma das autoras da pesquisa e coordenadora do projeto de extensão, realizou um treinamento com os alunos para nortear a coleta dos dados pertinentes das fichas de notificação compulsória das tentativas de suicídio, disponibilizadas pelo Núcleo de Vigilância Epidemiológica (NUVE) para arquivamento no NASF.

Foram incluídas na amostra as 55 fichas de notificação das ocorrências envolvendo pessoas de 12 a 25 anos de idade, classificadas como tentativa de suicídio no ano de 2015 , tanto por intoxicação exógena como por auto violência.

Os dados foram coletados por meio de consulta às fichas supracitadas, com auxílio de instrumento pré-elaborado, contendo informações sobre gênero, idade, cor/raça, estado civil, escolaridade, ocupação habitual, zona de habitação, local da ocorrência da tentativa, período do dia e mês do ano em que ocorreu a tentativa, meios de agressão, agentes tóxicos utilizados e reincidência.

As informações foram organizadas em um banco eletrônico de dados, estruturado no programa Excel do Microsoft Office. Em seguida, os dados foram analisados por meio de estatística descritiva, a partir dos cálculos das frequências absolutas e relativas das variáveis estudadas.

\section{Resultados}

Do total de 55 notificações de tentativas de suicídio entre adolescentes e jovens, no ano de 2015, constatou-se que, em relação ao gênero, 58,18\% dos casos ocorreram com pessoas do sexo feminino e $41,82 \%$ com pessoas do sexo masculino.

No tocante à faixa etária, evidenciou-se que a maior prevalência de tentativas de suicídio $(32,73 \%)$ ocorreu entre os adultos jovens, na faixa dos 22 aos 25 anos de idade e a menor prevalência dos 12 aos 15 anos $(14,54 \%)$, sinalizando que as taxas de tentativas de suicídio aumentaram de acordo com a idade da população estudada (Figura 1).

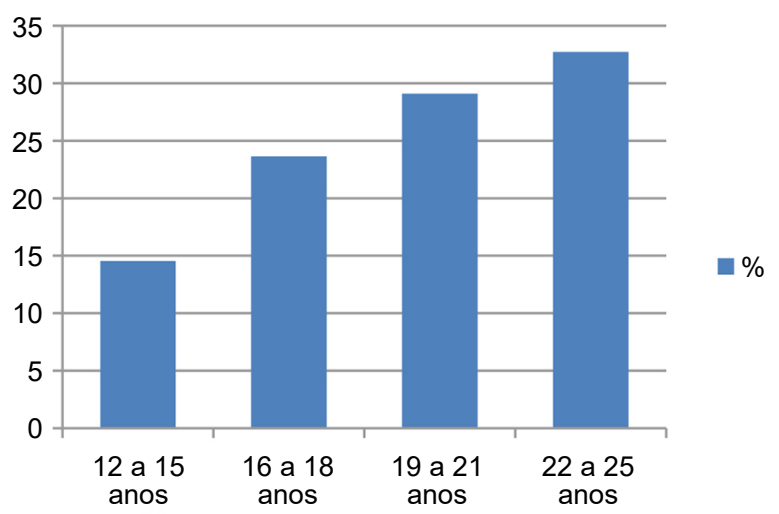

Figura 1 - Distribuição por idade de adolescentes e jovens que tentaram suicídio. Botucatu, SP, Brasil, 2015

Em relação à cor/raça dos adolescentes e jovens, $91,56 \%$ eram brancos, seguidos por $5,97 \%$ da raça negra e $2,47 \%$ da raça parda, conforme informações que compunham as fichas de notificação, preenchidas pelo médico responsável pelo caso. Já em relação ao estado civil, $80 \%$ eram solteiros, $16,37 \%$ mantinham união estável e em 3,63\% das fichas não constava esta informação.

Foram coletados dados em relação à escolaridade dessa população, que se apresentou do seguinte modo: $10,91 \%$ tinham fundamental II completo, $47,27 \%$ tinham fundamental II incompleto, $12,73 \%$ ensino médio completo, $25,45 \%$ ensino médio incompleto e $3,64 \%$ ensino superior incompleto.

As ocupações mais prevalentes dos adolescentes e jovens estudados foram estudantes (29,09\%) e donas de casa $(16,36 \%)$, entretanto, $23,64 \%$ eram desempregados (Figura 2).

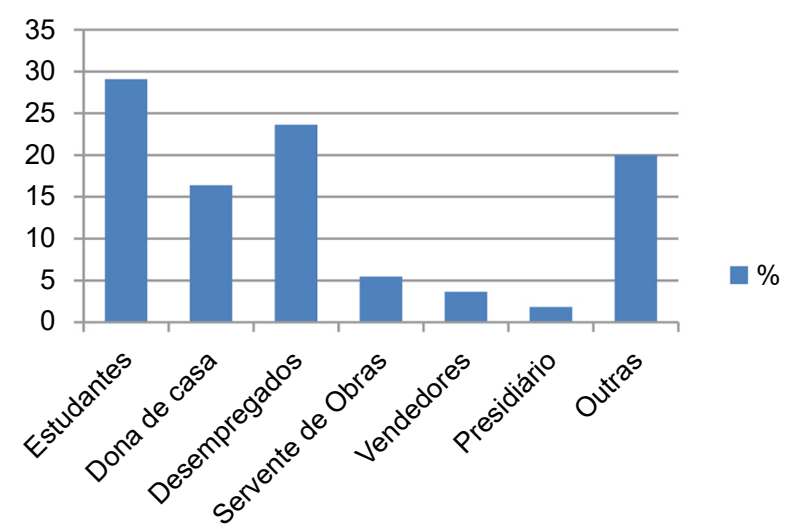

Figura 2 - Distribuição por ocupação de adolescentes e jovens que tentaram suicídio. Botucatu, SP, Brasil, 2015

Quanto à zona de habitação dos adolescentes e jovens, foi observado que $94,05 \%$ residiam na zona urbana e 5,95\% residiam na zona rural. Já em relação 
ao local de ocorrência das tentativas de suicídio, verificou-se que $92,72 \%$ aconteceram dentro da própria residência, 3,64\% em via pública, 1,82\% em habitação coletiva e outros locais de ocorrência não detalhados na ficha de notificação somam $1,82 \%$ dos casos.

Evidenciou-se que o período em que houve maior número de tentativas foi o período da noite com $41,82 \%$ dos casos registrados, seguidos por $27,27 \%$ no período da tarde, $20 \%$ no período da manhã e 10,91\% no período da madrugada.

Em relação ao mês em que ocorreu a tentativa de suicídio, constatou-se a seguinte distribuição: janeiro (20\%), fevereiro $(10,90 \%)$, março $(10,90 \%)$, abril $(5,46 \%)$, maio $(0 \%)$, junho $(7,28 \%)$, julho $(3,64 \%)$ agosto $(5,46 \%)$, setembro $(5,46 \%)$, outubro $(9,10 \%)$, novembro $(10,90 \%)$ e dezembro $(10,90 \%)$.

Os meios de agressão mais comumente utilizados foram o envenenamento ( $80 \%)$, a utilização de objeto perfuro cortante $(9,09 \%)$, o enforcamento $(5,45 \%)$ e outros (3,64\%). Em $1,82 \%$ dos casos não havia esta informação disponível na ficha de notificação.

Dentre as intoxicações exógenas (envenenamentos), em $78,18 \%$ das fichas de notificação não havia menção ao agente tóxico utilizado, em $20 \%$ dos casos foram utilizados medicamentos e em 1,82\% das ocorrências, os adolescentes e jovens fizeram uso de agrotóxicos.

No presente estudo, foram classificados como reincidentes $25,45 \%$ dos casos e como não reincidentes, $74,55 \%$ das ocorrências.

\section{Discussão}

As tentativas de suicídio são eventos complexos, envolvendo determinantes sociais, econômicos, culturais e biológicos(2,3,16). A experiência de passar por uma separação afetiva, a existência de conflitos familiares, a ansiedade e a depressão são fatores fortemente associados ao risco para suicídio(16-17).

Uma pesquisa que examinou os fatores preditores para tentativas de suicídio entre adolescentes com diagnóstico de depressão, na Coreia do Sul, evidenciou que quase metade dos adolescentes deprimidos já havia realizado tentativa de suicídio, sendo que destes, a maioria eram meninas(18). Apesar de não termos informações a respeito de diagnóstico de depressão nas fichas de notificação dos adolescentes que compuseram a amostra de nosso estudo, também observamos que a maior parte das ocorrências foi evidenciada entre pessoas do sexo feminino.

De acordo com os resultados da presente pesquisa, quanto maior a idade dos adolescentes e jovens, maiores as taxas de tentativas de suicídio. De modo geral, observa-se que as taxas de óbito por suicídio também se elevam com o aumento da idade ${ }^{(2,7)}$. Do mesmo modo, esta relação é apontada por outros autores ${ }^{(11)}$, ao afirmar a existência de maior prevalência do evento entre adolescentes mais velhos e adultos jovens.

É possível evidenciar o predomínio da raça branca entre os adolescentes e jovens, tanto na presente investigação, como em outros que buscaram caracterizar o perfil da população que tenta o suicídio(3).

A maioria dos adolescentes e jovens caracterizados em nosso estudo era composta por solteiros, estado civil esperado para esta faixa etária, no contexto estudado. No entanto, melhor detalhamento sobre esta questão, como a existência de um relacionamento afetivo, poderia contribuir para predizer o papel do suporte social para a prevenção das tentativas de suicídio nessa população.

A maior parte dos adolescentes e jovens estudados neste levantamento possuía ensino fundamental incompleto. Ademais, ressalta-se que a literatura associa a baixa escolaridade às tentativas de suicídio não somente entre adolescentes e jovens, mas também nas demais faixas etárias ${ }^{(3)}$.

Nossa pesquisa revelou maior proporção de estudantes, dada a idade dos adolescentes e jovens, mas também percentual considerável daqueles identificados como desempregados. Nesse sentido, considera-se importante destacar que a literatura já evidencia uma associação de fatores socioeconômicos como o desemprego e taxas de suicídio em adolescentes, na qual níveis mais altos de desemprego e desigualdade social foram associados a taxas mais altas de suicídio(19).

O estudo constatou que as maiores taxas de tentativas de suicídio entre adolescentes e jovens ocorrem na área urbana, na própria residência, assim como identificado em levantamento nacional dos casos de tentativas de suicídio notificados no Sinan, entre 2011 e $2016^{(3)}$.

Não localizamos até o momento outros estudos que retratassem o período do dia em que comumente ocorrem as tentativas de suicídio. A maior parte das tentativas entre adolescentes e jovens, nesta pesquisa, ocorreu no período noturno, possivelmente pelo fato de haver menor fluxo de pessoas em áreas comuns das residências, local de maior ocorrência dessas tentativas.

A pesquisa realizada sinaliza maior prevalência de tentativas de suicídio no mês de janeiro, período em que normalmente os adolescentes se encontram em férias escolares. Prevalências significantes também foram encontradas nos meses de fevereiro, março, novembro e dezembro, em igual proporção.

A maior parte das ocorrências levantadas nessa investigação se deu por envenenamento. Apesar de a maior parte das fichas de notificação compulsória não mencionar o agente tóxico utilizado para a tentativa de suicídio, em parte delas constava que foi por meio de ingestão de medicamentos e de agrotóxicos. Em relação a isso, convém destacar que a Organização Mundial da 
Saúde é enfática ao colocar que a restrição do acesso aos meios que o indivíduo poderia utilizar para atentar contra a própria vida, como agrotóxicos e armas de fogo, é uma das estratégias mais eficazes de prevenção do suicídio(2).

Ainda merece ser destacado o fato de mais de um quarto dos adolescentes e jovens serem classificados como reincidentes em relação às tentativas de suicídio, evento não discutido em literatura considerando essa faixa etária específica, mas que pode representar um prejuízo para a inovação e a economia da sociedade. De modo geral, mais de $31,3 \%$ das tentativas de suicídio entre as mulheres e $26,4 \%$ das tentativas entre os homens, tiveram caráter repetitivo, em levantamento realizado pelo Ministério da Saúde ${ }^{(3)}$.

De maneira geral, salienta-se como limitação da presente pesquisa a possibilidade de subnotificação das tentativas de suicídio entre adolescentes e jovens, bem como conteúdos deficientes referentes a algumas variáveis, como estado civil, local da ocorrência e meios de agressão, em que não havia a informação disponível em todas as fichas de notificação levantadas. Com a publicação da Política Nacional de Prevenção da Automutilação e do Suicídio, o poder público pretende fortalecer as notificações de casos suspeitos ou confirmados de violência autoprovocada, realizadas não somente pelos serviços de saúde, mas também por estabelecimentos de ensino(20).

O fenômeno da tentativa de suicídio entre adolescentes vem sendo melhor explorado nos últimos anos, sobretudo com enfoque para estudos epidemiológicos, que permitem compreensão do diagnóstico quantitativo do problema. Estudos que busquem aprofundar a compreensão sobre este evento podem ser realizados dando voz aos adolescentes, suas famílias e às equipes multiprofissionais, possibilitando intervenções de acordo com o contexto familiar e cultural e incrementando a formação profissional na área.

\section{Conclusões}

O perfil de adolescentes e adultos jovens que realizam tentativas de suicídio, no município estudado, caracteriza-se principalmente por mulheres, da raça branca, estudantes, solteiras, residentes em zona urbana, com tentativa de suicídio no próprio domicílio, por envenenamento, assemelhando-se ao perfil das tentativas entre adultos, apontado na literatura. Evidenciou-se ainda que, quanto maior a idade, maior a prevalência de tentativas de suicídio, sinalizando a necessidade de os profissionais programarem estratégias de prevenção inclusive entre crianças e adolescentes, a fim de se evitar, futuramente, não somente as tentativas, mas também o suicídio entre esses jovens. Para tal, é imprescindível que haja trabalho articulado em um sistema de redes de atenção à saúde dessa população, envolvendo a família, os profissionais da área da saúde, da educação, da assistência social e outros programas comunitários, de modo que a proteção à vida e à saúde de crianças, adolescentes e jovens seja um compromisso assumido por todos os segmentos da sociedade.

\section{Agradecimentos}

Ao Núcleo de Apoio à Saúde da Família pela disponibilidade dos arquivos para a coleta de dados.

\section{Referências}

1. Ministério da Saúde (BR). Proteger e cuidar da saúde dos adolescentes na atenção básica. [Internet]. Brasília (DF): Ministério da Saúde; 2017 [Acesso 5 abril 2019]. Disponível em: http://bvsms.saude.gov.br/bvs/ publicacoes/proteger_cuidar_adolescentes_atencao_ basica.pdf

2. World Health Organization. Preventing suicide: a global imperative. [Internet]. Geneve: World Health Organization; 2014 [cited 2016 May 17]. Available from: http://apps.who.int/iris/bitstream/ handle/10665/131056/9789241564779_eng. pdf?sequence $=1$

3. Ministério da Saúde (BR).Secretaria de Vigilância em Saúde. Perfil epidemiológico das tentativas e óbitos por suicídio no Brasil e a rede de atenção à saúde. Boletim Epidemiológico. [Internet]. Brasília (DF): Ministério da Saúde; 2017. [Acesso 5 abril 2019]. Disponível em: http://portalarquivos2.saude.gov.br/images/pdf/2017/ setembro/21/2017-025-Perfil-epidemiologico-dastentativas-e-obitos-por-suicidio-no-Brasil-e-a-rede-deatencao-a-saude.pdf

4. Ministério da Saúde (BR). Agenda de Ações Estratégicas para a Vigilância e Prevenção do Suicídio e Promoção da Saúde no Brasil. [Internet]. Brasília (DF): Ministério da Saúde; 2017. [Acesso 5 abril 2019]. Disponível em: http://www.saude.gov.br/images/ pdf/2017/setembro/21/17-0522-cartilha---AgendaEstrategica-publicada.pdf

5. Zhao S, Zhang J. Suicide Risks among Adolescents and Young Adults in Rural China. Int J Environ Res Public Health. 2015; 12(1):131-45. doi: 10.3390/ ijerph120100131

6. Shilubane HN, Robert AC, Ruiter Arjan ERB, Borne BVD, Shamagonam J, Reddy PS. Psychosocial Correlates of Suicidal Ideation in Rural South African Adolescents. Child Psychiatry Hum Dev. 2014; 45: 153-62. doi: 10.1007/s10578-013-0387-5.

7. Chau K, Kabuth B, Chau N. Gender and Family Disparities in Suicide Attempt and Role of Socioeconomic, School, and Health-Related Difficulties in Early 
Adolescence. Biomed Res Int. 2014; 314521. doi: 10.1155/2014/314521

8. Wyman PA. Developmental Approach to Prevent Adolescent Suicides. Am J Prev Med. 2014; 47(3): 2516. doi: 10.1016/j.amepre.2014.05.039

9. Rojas IG, Saavedra JE. Cohesión familiar e ideación suicida en adolescentes de la costa peruana en el año 2006. Rev Neuropsiquiatr. 2014; 77:250-61. doi: 10.20453/rnp.v77i4.2194

10. Silva L. Suicide among children and adolescents: a warning to accomplish a global imperative. Acta Paul Enferm. 2019; 32(3):III-IVI. doi: 10.1590/1982-

11. Patton GC, Coffey C, Romaniuk H, Mackinnon A, Carlin BJ, Degenhhardt $L$, et al. The prognosis of common mental disorders in adolescentes: a 14-year prospective cohort study. Lancet. 2014; 383: 1404-11. doi: 10.1016/S0140-6736(13)62116-9

12. Abreu N, Dias I, Cascais M, Luz A, Moleiro P. What are the most frequent diagnoses in adolescence? The reality of an Adolescent Medicine Clinic. Einstein. (São Paulo). 2018; 16(2): eA04225. doi: 10.1519/ s1679-45082018ao4225

13. You Z, Chen M, Yang S, Zhou Z, Qin P. Childhood Adversity, Recent Life Stressors and Suicidal Behavior in Chinese College Students. PLoS One. 2014; 9(3): 86672. doi: 10.1371/journal.pone.0086672

14. Claumann GS, Pinto AA, Silva DAS, Pelegrini A. Prevalência de pensamentos e comportamentos suicidas e associação com a insatisfação corporal em adolescentes. J Bras Psiquiatr. 2018; 67(1): 3-9. doi: 10.1590/0047-2085000000177

15. Silva L, Afonso BQ, Santos MR, Baliza MF, Rossato LM, Szylit R. Care for families after suicide loss: nursing academic experience. Rev Bras Enferm. 2018; 71(5): 2206-12. doi: 10.1590/0034-7167-2017-0445

16. Santos MSP, Silva TPS, Pires CMC, Ramos PGX, Sougey EB. Identificação de aspectos associados à tentativa de suicídio por envenenamento. J Bras Psiquiatr. 2017; 66(4): 197-202. doi: 10.1590/0047-2085000000171

17. Toro-Tobar RA, Grajales-Giraldo FL, Sarmiento-López JC. Riesgo suicida según la tríada cognitiva negativa, ideación, desesperanza y depresión. Aquichan. 2016; 16(4):473-86. doi: 10.5294/aqui.2016.16.4.6

18. Kwon A, Song J, Yook K, Jon D, Jung $M H$, Hong $\mathrm{N}$ et al. Predictors of suicide attempts in clinically depressed korean adolescents. Clin Psychopharmacol Neurosci. 2016; 14: 383-7. doi: 10.9758/cpn.2016.14.4.383

19. Jaen-Varas D, Mari J, Asevedo E, Borschmann R, Diniz E, Ziebold $C$, et al. The association between adolescent suicide rates and socioeconomic indicators in Brazil: a 10-year retrospective ecological study. Braz J Psychiatry. 2019. http://dx.doi.org/10.1590/1516-4446-2018-0223 20. Lei no 13.819, de 26 de abril de 2019 (BR). Institui a Política Nacional de Prevenção da Automutilação e do Suicídio. Diário Oficial da União, 9 de abril, Edição 81, Seção 1. p. 1. Brasília (DF); 2019. Disponível em: http:// www.in.gov.br/web/dou/-/lei-n\%C2\%BA-13.819-de-26de-abril-de-2019-85673796

\section{Contribuição dos autores}

Concepção e planejamento do estudo: Erasmo de Carvalho Oliveira e Lucía Silva. Obtenção dos dados: Erasmo de Carvalho Oliveira, Tatiane Scolastrici Meucci e Lucía Silva. Análise e interpretação dos dados: Erasmo de Carvalho Oliveira, Tatiane Scolastrici Meucci, Lisabelle Mariano Rossato, Ana Márcia Chiaradia Mendes Castillo e Lucía Silva. Análise estatística: Erasmo de Carvalho Oliveira, Tatiane Scolastrici Meucci e Lucía Silva. Redação do manuscrito: Erasmo de Carvalho Oliveira, Tatiane Scolastrici Meucci, Lisabelle Mariano Rossato, Ana Márcia Chiaradia Mendes Castillo e Lucía Silva. Revisão crítica do manuscrito: Lisabelle Mariano Rossato, Ana Márcia Chiaradia Mendes Castillo e Lucía Silva.

Todos os autores aprovaram a versão final do texto.

Conflito de interesse: os autores declararam que não há conflito de interesse.
Copyright $\odot 2020$ SMAD, Rev. Eletrônica Saúde Mental Álcool Drog. Este é um artigo de acesso aberto distribuído sob os termos da Licença Creative Commons CC BY-NC.

Esta licença permite que outros remixem, adaptem e criem a partir do seu trabalho para fins não comerciais, e embora os novos trabalhos tenham de lhe atribuir o devido crédito e não possam ser usados para fins comerciais, os usuários não têm de licenciar esses trabalhos derivados sob os mesmos termos. 